\title{
Expected Adverse Event
}

National Cancer Institute

\section{Source}

National Cancer Institute. Expected Adverse Event. NCI Thesaurus. Code C41333.

Any adverse event associated with a medical product or procedure, whose nature and severity have been previously observed, identified in nature, severity, or frequency, and documented in the investigator brochure, investigational plan, protocol, current consent form, scientific publication, or in other relevant and reliable document. The old term Side Effect is retired and should not be used. 